conclusions that factors other than birth asphyxia may be more important in etiology (see Progress in Pediatric Neurology III, PNB Publ, 1997;p377). The reliability of dried neonatal blood spot samples for immunoassay of inflammatory cytokines may permit a correlation between prenatal or perinatal infection and the development of childhood neurobehavioral disorders, including ADHD, often associated with subtle neurologic abnormalities.

Criteria for inclusion of cases in CP Registers are discussed in an article from the University of Western Australia (Badawi N, Stanley F et al. What constitutes cerebral palsy? Dev Med Child Neurol Aug 1998;40:520-527). As generally accepted, $\mathrm{CP}$ is a motor impairment resulting from brain pathology that is non-progressive and is manifested in early childhood. Exclusion criteria are those motor disorders caused by neurodegenerative diseases, neuromuscular disorders, spinal neural-tube defects, and brain tumors. The MRI and other laboratory investigations have permitted an etiological approach to classification of cases of CP that was formerly regarded as a "waste-basket" diagnosis.

\title{
ACUTE TRANSVERSE MYELITIS: CAUSES AND TREATMENT
}

Clinical manifestations, laboratory findings, management, and course of nine children diagnosed with acute transverse myelitis (ATM) between 1993 and 1996 are reported from the Children's University Hospital, Wurzburg, Germany. Spinal cord functions, including sensation, motor activity, and sphincter control, were affected in differing degrees, and neurologic symptoms and signs were often preceded by non-specific fever, nausea, and muscle pain. The peak of the illness and paraplegia was generally seen within 10 days and no later than 4 weeks. The thoracic spinal cord was principally involved in $80 \%$ of cases. Initially, tone and reflexes were decreased, and later, paralyses were associated with spasticity and hyperreflexia. CSF pleocytosis and/or elevated protein levels occurred in 4 patients. Peripheral nerve conduction velocity was normal, but muscle action potential amplitudes were decreased. An infectious causative agent was found in only 2 cases, with increasing antibody titers against echovirus 25 in one and Borrelia in one other. A para-, postinfective, postvaccinal myelitis was suspected in 7 cases. A review of the literature found bacterial, parasitic, and systemic lupus collagen disease as rare causes of ATM. A 3-day high-dose IV steroid pulse therapy ( $20 \mathrm{mg} / \mathrm{kg} /$ day prednisone) offered the most promising response in therapy. MRI excluded tumors, abscess, and vascular malformation. Multiple sclerosis, Guillain-Barre syndrome, and spinal cord infarction were more difficult to exclude. Of 6 patients followed up, 1 had a good recovery and 5 had a fair outcome. (Knebusch M, Strassburg HM, Reiners K. Acute transverse myelitis in childhood: nine cases and review of the literature. Dev Med Child Neurol September 1998;40:631-639). (Respond. Hans M Strassburg MD, Universitats Kinderklinik, Josef-Schneider-Str. 2 D-97080 Wurzburg, Germany).

COMMENT. Diagnostic criteria for acute transverse myelitis include: 1) acute paraplegia and sphincter disturbance with maximum impairment within 4 weeks; 2) bilateral segmental sensory impairment; 3) exclusion of spinal cord compression or systemic neurologic disease; and 4) consistent MRI and laboratory EMG findings. Prognosis is variable and residual sequelae are common.

\section{ASPERGILLUS MYELOPATHY}

Three children, ages 14 - 15 years, who developed myelopathy as the first manifestation of invasive aspergillosis are reported from the Children's Hospital Los Angeles, University of Southern California. All were immunosuppressed because of chemotherapy and antibiotics for treatment of leukemia. Symptoms 
presented with leg weakness and pain, followed in 2 to 6 days by loss of pain and temperature sensation, and finally complete myelopathy, with proprioceptive loss and urinary incontinence. One patient had brain involvement also, manifesting with delirium, spasticity, and arm weakness. Despite aggressive antifungal therapy, the patients died within 1 month of onset of symptoms. Postmortem findings showed multifocal meningeal exudates, cerebral and spinal hemorrhagic necrosis, and fungal abscess in the cord of one child, and aortic endarteritis, spinal necrosis and intramedullary hematoma in a second. A third, without postmortem, had shown CT and MRI evidence of a paravertebral mass with vertebral osteomyelitis, and a positive biopsy culture for Aspergillus. Blood and CSF cultures were negative. (Koh S, Ross LA, Gilles FH, Nelson MD Jr, Mitchell WG. Myelopathy resulting from invasive aspergillosis. Pediatr Neurol Aug 1998;19:135138). (Respond: Dr Susan Koh, Miami Children's Hospital, Neuroscience Center, 3100 SW 62nd Avenue, Miami, FL 33155).

COMMENT. Aspergillosis is a potential cause of myelopathy in immunosuppressed children treated with broad spectrum antibiotics.

\section{CEREBRAL CANDIDIASIS SEQUELAE IN PREMATURE INFANTS}

Cerebral candidiasis in two premature infants, severely handicapped on follow-up at 3 and 6 years, is reported from the Women's College Hospital, Toronto, Canada. Infant 1 had respiratory distress syndrome and was treated with ampicillin and gentamicin for 5 days. A Candida diaper rash with skin breakdown on day 12 was complicated by sepsis, treated with vancomycin and cefotaxime, and followed by systemic candidiasis, requiring amphotericin and 5-flucytosine. Head ultrasound showed echogenic fungal microabscesses in brain parenchyma that resolved after 37 days treatment. Follow-up at 6 years revealed mild cerebral palsy, severe aphasia, and ADHD. Infant 2 had respiratory distress syndrome, low Apgars, skin rash, sepsis treated with ampicillin, vancomycin and gentamicin, and at 6 days, candidiasis treated with amphotericin and flucytosine. Head ultrasound showed scattered echogenic microabscesses, and later, calcifications in the basal ganglia and periventricular areas. At 3 year follow-up, the child had spastic quadriplegia, speech impairment, and cognitive delay. (Scott PA, Ohlsson A. Sequelae associated with cerebral candidiasis in two premature infants. Acta PaediatrOct 1998;87:1090-2). (Respond: Dr PA Scott, NICU, Women's College Hospital, 76 Grenville Street, Toronto, Ontario, Canada M5S 1B2).

COMMENT. Previous reports of candidiasis involving the brain have involved autopsy descriptions of scattered microabscesses. Large doses of amphotericin $(1 \mathrm{mg} / \mathrm{kg} / \mathrm{d})$ and 5-flucytosine were thought to explain the survival of the two infants described above, although the resolution of infection did not prevent significant neurologic handicaps as sequelae. Treatment was continued for 37 days in case 1, with the better outcome, and 22 days in case 2, more severely handicapped. Perhaps more prolonged treatment may have improved the outcome, but serious side effects can be a problem. AAP 1997 Redbook recommends 6 weeks or longer treatment for systemic candidiasis in high risk patients.

\section{TOXOPLASMIC ENCEPHALITIS AND HYPER-IgM SYNDROME}

A 9-year-old boy with hyper-IgM syndrome complicated by impaired consciousness and disseminated toxoplasmosis is reported from Nagoya University School of Medicine, Japan. X-linked hyper-IgM syndrome was diagnosed at 5 months of age and treated with IV immunoglobulin. On admission, he had involuntary movements and convulsions. CT and MRI showed multiple lucencies 